\section{The human superior colliculus: Neither necessary, nor sufficient for consciousness?}

DOI: $10.1017 / \mathrm{S} 0140525 X 0700115 X$

\author{
Susanne Watkins and Geraint Rees \\ Wellcome Trust Centre for Neuroimaging and Institute for Cognitive \\ Neuroscience, University College London, London WC1 N 3AR, United \\ Kingdom. \\ s.watkins@fil.ion.ucl.ac.uk g.rees@fil.ion.ucl.ac.uk \\ http://www.fil.ion.ucl.ac.uk/ grees
}

\begin{abstract}
Non-invasive neuroimaging in humans permits direct investigation of the potential role for mesodiencephalic structures in consciousness. Activity in the superior colliculus can be correlated with the contents of consciousness, but it can be also identified for stimuli of which the subject is unaware; and consciousness of some types of visual stimuli may not require the superior colliculus.
\end{abstract}

Merker presents a wide-ranging overview in which a central role for the mesodiencephalic system in consciousness is proposed. Specifically, it is suggested that activity in the superior colliculus (SC) is necessary for changes in conscious content to occur, and activity in mesodiencephalic structures is sufficient to support consciousness. In humans, there is increasing evidence that activity in subcortical structures, such as the SC, can indeed be correlated with the contents of consciousness. Human SC is visually responsive in a retinotopic fashion (Schneider \& Kastner 2005; Sylvester et al. 2007), and Merker highlights our recent demonstration that changes in SC activity (accompanied by similar changes in activity in retinotopic early visual cortex) are correlated with altered perception in a visual illusion induced by sound (Watkins et al. 2006). Moreover, other subcortical structures anatomically adjacent and closely linked to the SC, such as the lateral geniculate nucleus, show fluctuations in activity closely correlated with changes in the contents of consciousness during binocular rivalry (Haynes et al. 2005; Wunderlich et al. 2005). But after damage to human primary visual cortex, SC activity can also be observed when moving visual stimuli are presented in a blind hemifield (Sahraie et al. 1997). Moreover, such SC activation can correlate with the emotional content of faces again presented in the blind hemifield (Morris et al. 2001). Such processing of subjectively invisible visual stimuli associated with SC activation can be associated with residual visual sensitivity (or "blindsight"; Weiskrantz 1997), which in turn may be related to different patterns of SC connectivity in patients with blindsight following hemispherectomy (Leh et al. 2006). Taken together, these data suggest that activation of the superior colliculus alone is therefore not sufficient for awareness, at least after damage to primary visual cortex.

The notion that activity in mesodiencephalic structures alone is insufficient to support consciousness is challenged by Merker's fascinating personal observations of the behavior of children with hydranencephaly. Despite these children apparently lacking most functioning cortical structures, a range of behaviors is reported that indicates some degree of limited responsiveness to their surroundings. However, caution is required before concluding that these individuals are conscious, and indeed, interpreting this as reflecting preserved mesodiencephalic function. Hydranencephaly describes a range of brain malformations that may vary with respect to time of onset, pathogenesis, and organization of any cortical remnants that may be present (Halsey 1987); and survival beyond six months is rare (McAbee et al. 2000). In the presently reported cases, the extent of cortical damage is unclear, so the extent to which any behaviors reflect mesodiencephalic structures alone in these individuals is not known. Moreover, responsiveness to the environment is a capacity exhibited by nearly any organism with a central nervous system, and cannot be unambiguously taken as a marker of consciousness. Verbal or manual reports are generally considered the primary criterion that can establish whether a percept is conscious (Weiskrantz 1997). Such behaviors, demonstrating intentionality, are not clearly evident in the present observations and many of the reported behaviors could be generated unconsciously or reflexively. This emphasizes both the difficulty in determining whether an individual unable or unwilling to give verbal or manual reports is conscious (Owen et al. 2006), and the consequent need to explore the possibility that non-invasive biomarkers of consciousness might be developed to permit such inference.

Three indirect lines of evidence also suggest that SC activation in humans may not be necessary, either, for changes in the contents of consciousness to occur. First, visual stimuli that stimulate only short-wave-sensitive cones (S-cones) in the retina are clearly visible (and indeed can influence attention and behavior; Sumner et al. 2006), even though the SC receives no direct projections from short-wave-sensitive cones and is therefore unlikely to be activated by such stimuli. Second, although SC damage in humans can cause lateralized visual neglect (Sprague 1996) and consequent failure to represent the contents of consciousness in one half of the space, bilateral damage does not eliminate awareness (Weddell 2004). Finally, direct intracranial stimulation of human visual cortex that bypasses geniculostriate and retinotectal pathways can result in conscious visual percepts (Lee et al. 2000), suggesting that subcortical activity may not be necessary for all types of awareness. Although all these lines of evidence are indirect, they raise the question of whether SC activity is strictly necessary for all types of conscious visual percept.

The picture that emerges, at least in humans, appears to be more complex than a simple identification of particular parts of the mesodiencephalic system with a single role as a necessary and sufficient "gatekeeper" for the contents of consciousness. Indeed, it seems unlikely that activity in any single area of the human brain will be sufficient for consciousness (Rees et al. 2002). The consistent association of changes in activity in SC (and other subcortical) structures with fluctuations in awareness thus suggests that they may play a role as part of a network of cortical and subcortical areas whose activity might represent a minimally sufficient substrate for the contents of consciousness; but further research is required.

\section{ACKNOWLEDGMENT}

This work was supported by the Wellcome Trust. 